\title{
Avaliação da dor na classificação de risco: conhecimento de estudantes de enfermagem
}

\author{
Pain evaluation in risk classification: knowledge of nursing students \\ Evaluación del dolor en la clasificación de riesgos: conocimiento de estudiantes de \\ enfermería
}

Cristiane Chaves de Souza ${ }^{1 *}$, José Victor Soares da Silva ${ }^{1}$, Isabela de Souza Santana ${ }^{1}$, Laís Sousa da Silva ${ }^{1}$, Luciene Muniz Braga ${ }^{1}$, Edimara Aparecida Buonicontro ${ }^{1}$, Luana Vieira Toledo ${ }^{1}$, Patrícia de Oliveira Salgado'.

\section{RESUMO}

Objetivo: Verificar o nível de conhecimento teórico de estudantes do curso de Enfermagem sobre a avaliação da dor durante a classificação de risco, utilizando como recurso à régua da dor. Métodos: Estudo transversal realizado com estudantes do último ano do curso de bacharelado em Enfermagem de uma universidade pública brasileira. Os dados foram coletados entre setembro e novembro de 2016, a partir de um questionário autorrespondido pelos alunos. Realizou-se análise estatística descritiva e inferencial. Resultados: A maioria dos estudantes (75,9\%) referiu obter, durante a graduação, conhecimento teórico necessário sobre a avaliação da dor para a prática clínica. Dentre os participantes, $62,1 \%$ conheciam a régua da dor e a utilizaram em algum momento durante as atividades práticas. A avaliação correta da dor esteve diretamente relacionada com a correta classificação de risco do usuário $(r=0,773 ; p<0,01)$. Conclusão: $O$ conhecimento dos estudantes de Enfermagem sobre a utilização da régua da dor foi satisfatório, e a maioria dos acadêmicos avaliam a dor na prática clínica e utilizam instrumentos nessa avaliação.

Palavras-chave: Enfermagem, Serviços médicos de emergência, Triagem, Medição da dor.

\begin{abstract}
Objective: To verify the level of theoretical knowledge of nursing students on pain assessment during risk classification, using the pain rule as a resource. Methods: Cross-sectional study conducted with students in the last year of the Bachelor of Nursing course at a Brazilian public university. Data were collected between September and November 2016, from a self-answered questionnaire by the students. Descriptive and inferential statistical analysis was performed. Results: Most students (75.9\%) reported obtaining, during graduation, necessary theoretical knowledge about pain assessment for clinical practice. Among the participants, $62.1 \%$ knew the rule of pain and used it at some point during practical activities. The correct pain assessment was directly related to the user's correct risk classification $(r=0.773 ; p<0.01)$. Conclusions: The knowledge of nursing students on the use of the pain rule was satisfactory, and most academics assess pain in clinical practice and use instruments in this assessment.
\end{abstract}

Keywords: Nursing, Emergency medical services, Triage, Pain measurement.

\section{RESUMEN}

Objetivo: Verificar el conocimientos teórico de los estudiantes del curso de enfermería sobre la evaluación del dolor durante la clasificación del riesgo, utilizando la regla del dolor como recurso. Métodos: Estudio transversal realizado con estudiantes del último año de la carrera de Licenciatura en Enfermería de una universidad pública brasileña. Los datos fueron recolectados entre septiembre y noviembre de 2016, a partir de un cuestionario auto-respondido por los estudiantes. Se realizó análisis estadístico descriptivo e inferencial. Resultados: La mayoría de los estudiantes $(75,9 \%)$ refirió haber obtenido durante la graduación los conocimientos teóricos necesarios sobre evaluación del dolor para la práctica clínica. Entre los participantes, el 62,1\% conocía la regla del dolor y la utilizó en algún momento durante las actividades prácticas. La evaluación correcta del dolor se relacionó directamente con la clasificación de riesgo correcta del usuario $(r=0,773 ; p<0,01)$. Conclusiones: El conocimiento de los estudiantes de enfermería sobre el uso de la regla del dolor fue satisfactorio y la mayoría de los académicos evalúan el dolor en la práctica clínica y utilizan instrumentos en esta evaluación.

Palabras clave: Enfermería, Servicios médicos de emergencia, Triaje, Medición del dolor.

${ }^{1}$ Universidade Federal de Viçosa (UFV), Viçosa - MG. *E-mail: souza.cris@gmail.com 


\section{INTRODUÇÃO}

A International Association for the Study of Pain (IASP) caracteriza a dor como uma experiência sensorial e emocional desagradável associada a uma lesão tecidual real ou potencial, ou descrita em termos de tal dano (IASP, 1994; NANDA-I, 2018). Ademais, outros estudos classificam-na como quinto sinal vital, sendo uma experiência somática refletora do temor de uma pessoa frente a ameaça à sua integridade física ou existencial. (COHEN M, et al., 2018; VIVEIROS WL, 2018).

A dor pode ter início súbito ou lento, intensidade leve à intensa, apresentando-se recorrentemente ou constantemente, com duração esperada, antecipada ou prolongada. Sendo sua cronicidade classificada em relação ao tempo de duração, quando menor que três meses é considerada como dor aguda e, quando maior que três meses, dor crônica (NANDA-I, 2018).

O manejo da dor é uma tarefa complexa, e a correta avaliação direciona para uma conduta terapêutica apropriada, permitindo acompanhar a qualidade das intervenções, eficácia dos tratamentos e dos cuidados (BATALHA LMC, 2016). Para isso, é necessária a aplicação de instrumentos que avaliem a dor do paciente, e que esses sejam de fácil aplicabilidade, a fim de otimizar o cuidado de enfermagem prestado ao paciente com dor (BERTONCELLO KCG, et al., 2016; LEITE ACS, et al., 2016).

Diversos protocolos são utilizados, a nível mundial, para classificação de risco, são eles o Sistema de Triagem de Manchester (STM), a Emergency Severity Índex (ESI), a Australasian Triage Scale (ATS) e o Canadian Triage Acuity Scale. Dentre esses, destaca-se o STM como principal protocolo utilizado no Brasil pelos enfermeiros na classificação de risco nos serviços de urgência. (SILVA PL, et al., 2016; CARMO BA e SOUZA G, 2017; FRANCO B, et al., 2018).

Dentre os atendimentos nos serviços de urgências, destacam-se as queixas álgicas como principal motivo de busca por estes serviços, sendo o enfermeiro o profissional mais indicado para avaliar, classificar o risco e determinar o nível de prioridade clínica do atendimento destes usuários (SILVA ATD, et al., 2018). Para facilitar a avaliação da dor permitindo resultados mais confiáveis foram elaborados instrumentos internacionalmente validados, unidimensionais de fácil, rápida aplicação e baixo custo, são elas a Escala Visual Analógica (EVA), Escala Numérica (EN), Escala Qualitativa (EQ) e a Escala de Faces (EF), capazes de avaliar a intensidade, a localização, as qualidades afetivas e sensoriais da dor (SILVA ATD, et al., 2018).

Em um estudo brasileiro, realizado com 43 profissionais de enfermagem de um hospital de urgência de Sergipe, $46,5 \%$ dos entrevistados não possuíam conhecimento sobre a existência de escala específica de avaliação da dor em crianças menores de três anos (SANTOS ACA, et al., 2017). Outro estudo brasileiro, de revisão integrativa da literatura evidenciou que os profissionais de enfermagem muitas vezes desconhecem ou possuem dificuldades em interpretar e utilizar as escalas de avaliação da dor, bem como o distanciamento do enfermeiro do processo de avaliação e gestão na utilização destas escalas (OLIVEIRA PEP, et al., 2016). A partir desses achados, percebe-se que a avaliação da dor ainda representa um grande desafio para 0 enfermeiro, sobretudo aos que realizam a classificação de risco em serviços de urgência, o que suscita a necessidade de ampliação dos conhecimentos sobre a avaliação da dor entre enfermeiros desde a sua formação.

De acordo com um estudo brasileiro, $65,2 \%$ dos estudantes de Enfermagem entrevistados relataram que a graduação foi insuficiente para adquirir os conhecimentos e informações em relação à dor para o tratamento (SOUSA NN e BENITO LAO, 2016). Tendo em vista a importância de conhecer o nível de preparo dos alunos do curso de bacharelado em Enfermagem de uma Universidade Pública Federal brasileira sobre a avaliação da dor na classificação de risco e adequar os conteúdos ministrados, colocou-se a seguinte questão de investigação: Qual é o conhecimento teórico de estudantes do último ano do curso de Enfermagem sobre a avaliação da dor, utilizando a régua da dor, em usuários atendidos na classificação de risco em serviços de urgência?

Assim, delineou-se a presente investigação com objetivo de verificar o nível de conhecimento teórico de estudantes do curso de Enfermagem sobre a avaliação da dor durante a classificação de risco, utilizando como recurso à régua da dor. 


\section{MÉTODOS}

Trata-se de estudo transversal realizado com 29 estudantes do último ano do curso de bacharelado em Enfermagem de uma Universidade Pública Federal. A população do estudo foi composta por todos os 30 alunos matriculados no último ano do curso de Enfermagem. Foram excluídos os estudantes que se recusaram a participar do estudo, totalizando um estudante.

A amostra final foi composta por 29 estudantes. Os dados foram coletados entre setembro e novembro de 2016 utilizando um questionário, autorrespondido pelos estudantes, em data e horário previamente agendados pelo pesquisador.

O instrumento de coleta de dados foi composto por três partes: Parte 1 - orientações gerais para 0 preenchimento do questionário; Parte 2 - dados sobre o perfil acadêmico do estudante (idade, sexo, envolvimento com atividades de ensino, pesquisa e extensão, desenvolvimento de estágios extracurriculares) e sua percepção sobre a importância da avaliação da dor, mensurada a partir de uma escala do tipo Likert (1 = discordo totalmente; 2 = discordo; 3 = não concordo, nem discordo; 4 = concordo; 5 = concordo totalmente); Parte 3 - régua da dor e seis casos clínicos fictícios, validados em estudo anterior (SOUZA CC, 2016), refletindo situações reais do serviço de urgência para avaliação da dor.

Nessa parte do questionário, o estudante avaliou os casos clínicos e indicou o nível de dor a partir da régua da dor e a classificação de risco do usuário descrito no caso. A régua da dor classifica a severidade da dor em três níveis: leve (pontuação de 1 a 4), moderada (pontuação de 5 a 7) e intensa (pontuação de 8 a 10).

Em relação à classificação de risco, o STM permite a classificação em cores conforme o nível de dor, sendo: azul (dor leve a mais de sete dias), verde (dor leve a menos de sete dias), amarelo (dor moderada) e laranja (dor intensa) (SEBASTIÃO SMFA, 2019).

Considerou-se como conhecimento satisfatório acerca da régua da dor uma pontuação média maior ou igual a 3,6, ou seja, no mínimo $60 \%$ de acerto. Este ponto de corte foi estabelecido pela pontuação mínima necessária para aprovação do estudante nas disciplinas do curso de Enfermagem na Universidade estudada.

Os dados foram analisados utilizando o programa Excel versão 2010 por meio de estatística descritiva com tabelas de distribuição de frequência, medidas de tendência central (média e mediana) e de dispersão (desvio-padrão). Para análise inferencial, utilizou-se o programa estatístico Statistical Package for the Social Sciences (SPSS) versão 21,0.

Para verificar a existência de associação entre variáveis do perfil acadêmico e o número de acertos na indicação do nível de dor, realizou-se o Teste do Qui-Quadrado. Para investigar a associação entre a percepção do estudante sobre a avaliação da dor e o número de acertos na indicação do tipo de dor, utilizouse o teste de correlação de Spearman.

Para verificar a existência de associação entre as variáveis "número de acertos no tipo de dor" e "número de acertos na indicação do nível de risco" utilizou-se o teste de correlação de Pearson. Para todas as análises inferenciais, utilizou-se o nível de significância de 5\%. A investigação atendeu os preceitos éticos para pesquisas envolvendo seres humanos e foi aprovado pelo Comitê de Ética em Pesquisa da Universidade onde o estudo foi realizado (Parecer № 1.668.549).

\section{RESULTADOS}

Dos 29 estudantes participantes, a maioria foi do sexo feminino (89,7\%). A média de idade foi de 25 anos ( $\pm 4,7$ anos; $21-48$ anos). A maior parte dos estudantes participou de projetos de extensão $(96,6 \%)$ e ligas acadêmicas (62,1\%) (Tabela 1). 
Tabela 1 - Participação dos estudantes de Enfermagem em atividades extracurriculares durante a formação acadêmica. 2016. ( $\mathrm{N}=29)$.

\begin{tabular}{ccc}
\hline Atividades Extracurriculares & $\mathbf{n}$ & $\%^{*}$ \\
\hline Estágio Extracurricular & 10 & 34,5 \\
Projetos de Extensão & 28 & 96,6 \\
Projetos de Iniciação Científica & 11 & 37,9 \\
Ligas Acadêmicas & 18 & 62,1 \\
\hline
\end{tabular}

Legenda: *A percentagem não corresponde a 100\% porque essa variável apresenta múltiplas respostas.

Fonte: Souza CC, et al., 2020.

$\mathrm{Na}$ análise da existência de correlação entre a atividade extracurricular desenvolvida e o número de acertos do estudante na classificação da dor não foi evidenciada associação significativa entre estas variáveis de acordo com o teste do qui-quadrado ( $p>0,05)$.

Frente à avaliação da dor na prática clínica, os dados relativos à avaliação da intensidade e localização da dor foram os mais citados pelos estudantes, com percentual superior a $60,0 \%$ e a caracterização da dor 0 item menos citado (17,2\%) (Tabela 2).

Tabela 2 - Dados avaliados pelos estudantes de Enfermagem aquando da queixa de dor. 2016. ( $\mathrm{N}=29)$.

\begin{tabular}{ccc}
\hline Dados avaliados & $\mathbf{n}$ & $\%^{*}$ \\
\hline Intensidade & 23 & 79,3 \\
Localização & 18 & 62,1 \\
Paliativo e/ou Provocativo & 13 & 44,8 \\
Duração & 12 & 41,4 \\
Irradiação & 10 & 34,5 \\
Expressão Facial & 10 & 34,5 \\
Início & 10 & 34,5 \\
Relato de dor & 6 & 20,7 \\
Alterações sistêmicas e/ou nos sinais vitais & 6 & 20,7 \\
Outros & 6 & 20,7 \\
Caracterização & 5 & 17,2 \\
\hline
\end{tabular}

Legenda: *A percentagem não corresponde a 100\% porque essa variável apresenta múltiplas respostas.

Fonte: Souza CC, et al., 2020.

A maioria dos estudantes $(89,7 \%)$ conhece os instrumentos utilizados na prática clínica para avaliar a dor, sendo a EVA a mais citada por eles (76,9\%). Além disso, $62,1 \%$ dos estudantes afirmaram conhecer e já ter utilizado a régua da dor. Apenas $11,5 \%$ conhecem duas ou mais escalas para avaliação da dor.

De acordo com os resultados, $75,9 \%$ dos estudantes afirmaram que o conhecimento teórico sobre a avaliação da dor recebido durante o curso de Enfermagem foi importante para prepará-los para a prática clínica. A maioria dos estudantes $(62,1 \%)$ se sente seguro para avaliar a dor no contexto assistencial. No entanto, apenas $34,4 \%$ sabem utilizar com segurança a régua da dor. Para $100,0 \%$ dos estudantes a avaliação da dor é um cuidado importante para ser realizado pelo enfermeiro, sobretudo para aqueles que trabalham em serviço de urgência (Tabela 3). 
Tabela 3 - Perceção dos estudantes de Enfermagem acerca da avaliação da dor. 2016. (N=29).

\begin{tabular}{|c|c|c|c|c|c|c|c|c|c|c|}
\hline \multirow{2}{*}{ Itens da perceção dos estudantes } & \multicolumn{2}{|c|}{$1^{*}$} & \multicolumn{2}{|c|}{$2^{*}$} & \multicolumn{2}{|c|}{$3^{*}$} & \multicolumn{2}{|c|}{$4^{*}$} & \multicolumn{2}{|c|}{$5^{\star}$} \\
\hline & $\mathbf{N}$ & $\%$ & $\mathbf{N}$ & $\%$ & $\mathbf{n}$ & $\%$ & $\mathbf{n}$ & $\%$ & $\mathrm{n}$ & $\%$ \\
\hline $\begin{array}{l}\text { - Aprender a avaliar a dor é importante } \\
\text { para o enfermeiro, sobretudo para } \\
\text { aqueles que irão atuar no serviço de } \\
\text { urgência. }\end{array}$ & 0 & 0 & 0 & 0 & 0 & 0 & 3 & 10,3 & 26 & 89,7 \\
\hline $\begin{array}{l}\text { - Me sinto seguro para avaliar a dor na } \\
\text { prática clínica, sobretudo em serviços } \\
\text { de urgência. }\end{array}$ & 1 & 3,5 & 2 & 6,9 & 8 & 27,6 & 13 & 44,8 & 5 & 17,2 \\
\hline $\begin{array}{l}\text { - O conhecimento teórico recebido } \\
\text { durante a graduação me preparou } \\
\text { para atuar como enfermeiro na prática } \\
\text { clínica no que concerne à avaliação. }\end{array}$ & 0 & 0 & 2 & 6,9 & 5 & 17,2 & 15 & 51,7 & 7 & 24,2 \\
\hline $\begin{array}{l}\text { - Sei utilizar com segurança a régua } \\
\text { da dor. }\end{array}$ & 4 & 13,8 & 6 & 20,7 & 9 & 31,1 & 5 & 17,2 & 5 & 17,2 \\
\hline $\begin{array}{l}\text { - A régua da dor é um instrumento de } \\
\text { fácil aplicação na prática clínica. }\end{array}$ & 1 & 3,4 & 0 & 0 & 8 & 27,6 & 14 & 48,3 & 6 & 20,7 \\
\hline $\begin{array}{l}\text { - A utilização de um instrumento para } \\
\text { avaliar a dor facilita a mensuração } \\
\text { desta queixa no paciente. }\end{array}$ & 0 & 0 & 0 & 0 & 0 & 0 & 8 & 27,6 & 21 & 72,4 \\
\hline $\begin{array}{l}\text { - A dor é um fenômeno subjetivo, e } \\
\text { portanto, não necessita de } \\
\text { instrumentos para sua avaliação. }\end{array}$ & 16 & 55,2 & 9 & 31,0 & 4 & 13,8 & 0 & 0 & 0 & 0 \\
\hline
\end{tabular}

Legenda: *Avaliação do estudante: 1 (Discordo totalmente), 2 (Discordo), 3 (Não concordo, nem discordo), 4 (Concordo), 5 (Concordo totalmente).

Fonte: Souza CC, et al., 2020.

De acordo com o teste de correlação de Spearman, não se verificou associação $(p>0,05)$ entre a percepção dos estudantes sobre a avaliação da dor e o número de acertos na estratificação correta do tipo de dor (leve, moderada ou intensa).

Em relação aos acertos obtidos a partir da utilização da régua da dor na avaliação dos casos clínicos verificou-se que a média de acertos para discriminação desta queixa foi de 4,79 ( $\pm 1,08$; Mediana:5). A média de acertos na indicação correta do nível de risco do paciente a partir da classificação da dor foi de $3,97( \pm 1,65$; Mediana:4). O teste de correlação de Pearson mostrou haver uma associação diretamente proporcional entre as variáveis "Acertos na discriminação do tipo de dor", e "Acertos na classificação de risco" $(r=0,773 ; p<0,01)$.

\section{DISCUSSÃO}

Neste estudo, investigou-se a participação dos alunos em atividades extracurriculares durante a formação profissional. Dos 29 respondentes, 28 (96,6\%) envolveram-se em alguma atividade extracurricular. Contudo, cabe ressaltar que nenhuma das atividades de extensão e pesquisa desenvolvidas tinham foco no estudo da dor.

Semelhantemente, estudos apontam que a participação de alunos em atividades extracurriculares de enfermagem variam de $81,6 \%$ a $86,0 \%$ (BARBOSA ACS, et al., 2019; OLIVEIRA PMC, 2017). Observa-se que $o$ acadêmico de Enfermagem se preocupa em participar de atividades que proporcionam 0 desenvolvimento de competências em diferentes contextos.

Sempre que presente, a queixa de dor deve ser avaliada pelos enfermeiros que prestam cuidados em serviços de urgência, especialmente na classificação de risco. No presente estudo, $89,7 \%$ dos estudantes concordaram totalmente que é importante o enfermeiro avaliar a dor, sobretudo nesses serviços. 
Estudo realizado em um serviço de urgência de um hospital central da região centro de Portugal evidenciou que aproximadamente $55,3 \%$ dos usuários doentes foram triados por um discriminador de dor. Portanto, é necessário que o enfermeiro possua conhecimento sobre a avaliação da dor e das escalas utilizadas para mensurá-la na prática clínica (ANTÓNIO CAS, 2017).

$\mathrm{Na}$ amostra estudada, somente os dados de intensidade e localização da dor foram citados por mais de $50,0 \%$ dos estudantes como utilizados para avaliar a queixa da dor na prática clínica. Essas informações também foram apontadas, pela equipe multiprofissional de um hospital público brasileiro, como as frequentemente utilizadas no registro da avaliação da dor (NASCIMENTO LA, et al., 2016).

Vale salientar que a qualidade da avaliação da dor depende de outros itens fundamentais como a avaliação do que o indivíduo estava fazendo no momento que iniciou a dor, localização, duração, frequência, qualidade, intensidade, radiação para outros locais, características da dor, fatores provocativos e paliativos e impactos nas atividades de vida diária (ANTÓNIO CAS, 2017).

Neste estudo a maioria dos estudantes afirmou conhecimentos teóricos adquiridos durante a graduação os instrumentalizam para a avaliação da dor na prática clínica, tornando-os seguros para a sua execução. Corroborando com os dados, um estudo demonstrou que $51,0 \%$ dos enfermeiros que prestam cuidados em Unidades de Terapia Intensiva Neonatal, de seis hospitais públicos brasileiros, concordaram que o enfermeiro possui conhecimento suficiente para avaliar a dor do recém-nascido (COSTA T, et al., 2017).

Em contrapartida, estudo realizado com 247 estudantes de enfermagem de uma instituição de ensino privada da cidade de Brasília demonstrou que a maioria $(65,20 \%)$ não recebeu informações suficientes para realizar o manejo de pacientes com dor durante a graduação (SOUSA NN e BENITO LAO, 2016).

Neste contexto, um estudo reflexivo baseado na literatura científica revelou que em países em desenvolvimento, os profissionais da saúde durante a formação não receberam conhecimentos específicos acerca do manejo da dor, somando $91,0 \%$ de relatos sobre a fragilidade do ensino deste conteúdo (FERRARI MFM, et al., 2019).

Ainda corroborando, outro estudo realizado em um Hospital Universitário da Universidade Federal de Sergipe, com 82 profissionais de saúde, evidenciou o desconhecimento destes na diferenciação entre avaliação e mensuração da dor, uma vez que $57,3 \%$ afirmaram que a avaliação é realizada através de escalas (numérica, analógica e descritores verbais) e 63,4\% afirmaram que a mensuração é realizada pelos mesmos métodos (RIBEIRO MCO, et al., 2015).

A utilização de instrumentos para avaliação da dor é importante para melhorar a compreensão e para diminuir o viés da subjetividade do avaliador (SILVA TCR, 2017). Na presente investigação 89,7\% dos estudantes relataram conhecer instrumentos para avaliar a dor na prática clínica, sendo a EVA e a Régua da Dor conhecidas por $76,9 \%$ e $62,1 \%$ dos participantes, respectivamente. A maioria dos alunos concordou totalmente $(72,4 \%)$ que a utilização de um instrumento para avaliar a dor facilita a mensuração desta queixa.

A média geral de pontuação de acertos na indicação correta do nível de dor aponta que o conhecimento dos estudantes sobre a régua da dor é satisfatório. No entanto, apenas $34,4 \%$ concordaram que sabem utilizar com segurança esse instrumento.

Este fato chama atenção para a necessidade de as instituições de ensino proporcionarem aos alunos, durante o curso de Enfermagem, experiências clínicas em ambientes reais ou em práticas simuladas em laboratórios, para propiciar vivências na aplicação de instrumentos de avaliação da dor, em especial a régua da dor, no contexto da classificação de risco, de modo a aumentar a autoconfiança do aprendiz na utilização desta ferramenta na prática clínica.

Destaca-se ainda que não foram encontrados, até o momento, estudos que tenham avaliado o conhecimento teórico e prático de estudantes do curso de enfermagem, nem de enfermeiros, acerca do uso da régua da dor em nenhum cenário assistencial. 
Pesquisa realizada com 51 enfermeiros de Unidades de Terapia Intensiva Neonatal, em seis hospitais públicos de Curitiba e Região Metropolitana, evidenciou que $86,3 \%$ dos profissionais concordam que a utilização de escalas para a avaliação da dor é importante na prática clínica. Porém, somente $20,4 \%$ dos profissionais utilizavam sempre escalas para avaliação da dor em recém-nascidos (COSTA T, et al., 2017).

De maneira semelhante, outro estudo realizado com 164 enfermeiros, atuantes em um Serviço de Urgência de Portugal, identificou que $84,0 \%$ dos participantes relataram monitorar a dor do paciente através de escalas de dor sempre ou frequentemente, e 6,7\% disseram nunca utilizar essas escalas ou não as conhecerem (ANTÓNIO CAS, 2017).

Ainda nesse sentido, um estudo realizado em uma instituição de ensino privada de Aracajú, Sergipe, com 169 estudantes do último ano da graduação em enfermagem, mostrou que $88,2 \%$ e $88,8 \%$ dos discentes relataram aprendizagem acerca de escalas de dor em crianças e em adultos, respectivamente.

Porém, entre esses mesmos alunos, durante o estágio curricular, $77,5 \%$ disseram não encontrar o uso dessas escalas nos prontuários e somente $30,2 \%$ relataram já ter utilizado escalas para avaliar a dor dos pacientes. Além disso, apenas $25,4 \%$ e $59,1 \%$ dos discentes evidenciaram aptidão e segurança em realizar essa avaliação em crianças e em adultos, respectivamente (SANTOS AF, et al., 2019).

Assim, a partir da literatura disponível, percebe-se que apesar de os estudantes terem sido informados sobre o assunto, muitos ainda não se sentem completamente preparados para aplicar esse tipo de avaliação na prática clínica e grande parte dos profissionais da área também não fazem uso dessa ferramenta de auxílio à mensuração da dor.

Portanto, faz-se necessário o aprimoramento desse conhecimento durante a graduação e a continuidade desse ensino após a formação para uma adequada utilização de escalas para avaliação da dor, em especial o uso da régua dor em serviços de urgência.

A dor é a queixa mais frequente no setor de urgência e o tipo de dor é um dos discriminadores utilizados na classificação de risco no Sistema de Triagem de Manchester (DEUS GA, et al., 2018; SILVA MOF, 2017). Na presente investigação, as variáveis "Acertos no tipo de dor" e "Acertos na classificação de risco" foram diretamente associadas $(r=0,773 ; p<0,01)$, demonstrando que a correta avaliação da dor está relacionada com uma correta classificação de risco do usuário para o atendimento médico.

Resultado semelhante foi encontrado num estudo brasileiro realizado em 2016, demonstrando que a indicação correta do discriminador explica $77,0 \%$ dos acertos no nível de risco do usuário (SOUZA CC, et al., 2018). Tal fato, reforça que a correta avaliação da dor em serviços de urgência é imprescindível para a segurança do usuário, uma vez que o atendimento se inicia com uma correta indicação do nível de risco.

Além disso, segundo estudo realizado com 96 enfermeiros em Portugal, há uma associação positiva entre os bons conhecimentos na categorização da dor e a gestão da mesma durante o cuidado com o paciente, corroborando para a segurança e eficácia do tratamento do desconforto (MOTA M, et al., 2020).

Cabe ressaltar que existem lacunas na literatura científica acerca da competência clínica do enfermeiro e de estudantes de enfermagem sobre o uso da régua da dor na prática, sobretudo na classificação de risco utilizando o STM. Assim, identifica-se a necessidade de oportunizar ao aprendiz experiências práticas durante a formação acadêmica relacionadas aos conteúdos específicos sobre a dor, sua avaliação e controle.

Espera-se que estas vivências contribuam para a autoconfiança do estudante na avaliação da dor. Embora a presente investigação tenha utilizado casos clínicos, que refletem situações reais do serviço de urgência, apresenta como limitação o fato de ter sido dado foco apenas ao conhecimento teórico dos estudantes. Assim, sugere-se a realização de outros estudos com análise no conhecimento prático, bem como a autoconfiança do estudante para utilização da régua da dor na classificação de risco. 


\section{CONCLUSÃO}

O conhecimento teórico dos estudantes de Enfermagem sobre a utilização da régua da dor na classificação de risco dos usuários em serviço de urgência foi satisfatório. A maioria dos estudantes afirmou que executa a avaliação da dor na prática clínica, conhece os instrumentos utilizados para esse fim, sente-se seguro durante essa avaliação e considera satisfatório o conhecimento específico obtido durante o curso de Enfermagem. A correta classificação do paciente é o primeiro passo para garantir a segurança do usuário em serviços de urgência, pois a triagem para níveis de maior ou menor gravidade influencia diretamente no tempo de espera para atendimento médico e no gerenciamento dos recursos assistenciais necessários ao atendimento.

\section{REFERÊNCIAS}

1. ANTÓNIO CAS. Gestão da dor no Serviço de Urgência: práticas dos enfermeiros. Dissertação (Mestrado em Enfermagem MédicoCirúrgica). Escola Superior de Enfermagem de Coimbra, Coimbra, 2017; 152p.

2. BARBOSA ACS, et al. Profile of nursing graduates: competencies and professional insertion. Revista Latino-Americana de Enfermagem, 2019; 27: e3205.

3. BATALHA LMC, Avaliação da dor: manual de estudos - versão 1. Escola Superior de Enfermagem de Coimbra, 2016; 45p.

4. BERTONCELLO KCG, et al. Dor Aguda na Emergência: Avaliação e Controle com o Instrumento de MacCaffery e Beebe. J. Health Sci, 2016; 18(4): 251-256.

5. CARMO BA, SOUZA G. Atuação do enfermeiro na classificação de risco através do protocolo de manchester: uma revisão da literatura. Revista Eletrônica Acervo Saúde, 2018; Especial: S1081-S1088.

6. COHEN M, et al. Reconsidering the International Association for the study of pain definition of pain. Pain Reports, 2018 ; 3(2): e634.

7. COSTA T, et al. Nurses' knowledge and practices regarding pain management in newborns. Revista Da Escola de Enfermagem da USP, 2017; 51: e03210.

8. DEUS GA, et al. Acolhimento com avaliação e classificação de risco em um pronto socorro: estudo comparativo. Arquivos de Ciência da Saúde, 2018; 25(2): 20-23.

9. FERRARI MFM, et al. A dor como quinto sinal vital, desafios para a incorporação na formação em saúde. Revista Mineira de Enfermagem, 2019; 23: e1233.

10. FRANCO B, et al. Associações entre discriminadores do Sistema de Triagem de Manchester e diagnósticos de enfermagem. Revista Gaúcha de Enfermagem, 2018; 39: e2017-0131.

11. GRUPO BRASILEIRO DE CLASSIFICAÇÃO DE RISCO. Autenticidade do certificado do curso sistema Manchester de classificação de risco EAD e presencial - Grupo Brasileiro de Classificação de Risco. Belo Horizonte, 2015.

12. HERDMAN TH, KAMITSURU S. Diagnósticos de Enfermagem da NANDA-I: Definições e Classificação 2018-2020. 11nd ed. Porto Alegre: Artmed, 2018; 1187p.

13. INTERNATIONAL ASSOCIATION FOR THE STUDY OF PAIN. 2012. In: Part III: Pain Terms, A Current List with Definitions and Notes on Usage. Seattle. Disponível em: https://web.archive.org/web/20150113000208/http://www.iasp-pain.org/Taxonomy\#Pain. Acesso em: 25 jul. 2020.

14. LEITE ACS, et al. Acute chest pain intensity in a cardiopulmonary emergency unit. Revista Dor, 2016; 17(3): 159-63.

15. MOTA M, et al. Gestão da dor na prática de enfermagem no serviço de urgência. Millenium, 2020; 2(ed espec nำ5): $269-279$.

16. NASCIMENTO LA, et al. Pain management: evaluation of practices adopted by health professional of a secondary public hospital. Revista Dor, 2016; 17(2): 76-80.

17. OLIVEIRA PEP, et al. A enfermagem no manejo da dor em unidades de atendimento de urgência e emergência. Revista Eletrônica de Enfermagem, 2016. 18: e1171.

18. OLIVEIRA PMC. Perfil do egresso do curso de graduação de enfermagem de uma faculdade privada da região norte do Ceará. Dissertação (Mestrado em Saúde da Família) - Universidade Federal do Ceará - Campus Sobral - CE. 2017. 87p.

19. RIBEIRO MCO, et al. Knowledge of health professionals about pain and analgesia. Revista Dor, 2015; 16(3): $204-209$.

20. SANTOS ACA, et al. Conhecimento dos profissionais de enfermagem sobre as escalas de avaliação da dor em pediatria. Journal of Health connections, $2017 ; 1(1): 19-32$.

21. SANTOS AF, et al. Formação dos discentes de enfermagem acerca da avaliação da dor. Revista de Enfermagem UFPE On Line, 2019; 13(5): 1380-1386.

22. SEBASTIÃO SMFA. Marcadores de Risco dos Clientes admitidos no Serviço de Urgência. Dissertação (Mestrado em Enfermagem Médico-Cirúgica). Escola Superior de Saúde de Viseu, Viseu, 2019. 105p.

23. SILVA ATD, et al. Avaliação e intervenção da enfermagem frente ao paciente com dor crônica. Centro Universitário Uniceplac, 2018.

24. SILVA MOF. Avaliação e registo da dor no serviço de urgência: que realidade?. Dissertação (Mestrado em Enfermagem MédicoCirúgica). Escola Superior de Enfermagem de Coimbra, Coimbra, 2017. 146p.

25. SILVA PL, et al. Acolhimento com classificação de risco do serviço de Pronto-Socorro Adulto: satisfação do usuário. Revista da Escola de Enfermagem da USP, 2016; 50(3): 427-432.

26. SILVA TCR. Adaptação transcultural da escala multidimensional de avaliação da dor. Tese (Doutorado em Ciências). Escola de Enfermagem de Ribeirão Preto da USP, Ribeirão Preto, 2017. 191p.

27. SOUSA NN, BENITO LAO. Conhecimento de graduandos de enfermagem sobre a dor. Centro Universitário de Brasília, 2016.

28. SOUZA CC. Análise da confiabilidade do Sistema de Triagem de Manchester para determinar o grau de prioridade de pacientes em serviços de urgência. Tese (Doutorado em Enfermagem) - Escola de Enfermagem. Universidade Federal de Minas Gerais, Belo Horizonte, 2016;

29. SOUZA CC, et al. Reliability analysis of the manchester triage system: Inter-observer and intra-observer agreement. Revista LatinoAmericana de Enfermagem, 2018; 26: e3005

30. VIVEIROS WL, et al. Pain in emergency units: correlation with risk classification categories. Revista Latino-Americana de Enfermagem, 2018; 26: e3070. 136 p. 